\title{
Comparative matched-pair cohort analysis of the short-term clinical outcomes of mesenchymal stem cells versus hyaluronic acid treatments through intra-articular injections for knee osteoarthritis
}

Yong Sang Kim, Dong Suk Suh, Dae Hyun Tak, Pill Ku Chung, Yoo Beom Kwon, Tae Yong Kim and Yong Gon Koh ${ }^{*}$ (D)

\begin{abstract}
Purpose: Intra-articular injection of hyaluronic acid (HA) has shown promises in reducing pain and improving physical function in knee osteoarthritis (OA). Recently, cell-based therapies using mesenchymal stem cells (MSCs) have emerged as potential treatments. However, few studies have compared the treatment outcomes between MSCs and HA. This study aimed to compare the clinical and radiological outcomes of intra-articular injections of MSCs versus HA in patients with knee OA.

Methods: A cohort of 209 patients with knee OA were retrospectively screened for those who underwent intraarticular injections using MSCS or HA. Thirty MSC-treated patients (MSC group) were pair-matched with thirty HAtreated patients (HA group) based on gender and age. Clinical outcomes were evaluated using the visual analog scale (VAS), International Knee Documentation Committee (IKDC) rating system, and Lysholm scoring system. Radiological evaluation was assessed using the Kellgren-Lawrence (K-L) grading system.

Results: MSC treatment yielded consistent significant improvements in VAS, IKDC and Lysholm scores. In the HA group, VAS scores significantly decreased at 1 month, slightly increased at 3 months, and increased significantly from 3 months to 1 year after injection. The IKDC and Lysholm scores improved significantly until 3 months, but gradually worsened thereafter. Significantly greater improvements in VAS $(P=0.041)$, IKDC $(P=0.014)$, and Lysholm $(P=0.020)$ scores were observed in the MSC group compared to those in the HA group at 1-year post-treatment. The K-L grade worsened in a few patients, especially those in the HA group, albeit no significant difference.
\end{abstract}

Conclusions: MSC group showed better VAS, IKDC, and Lysholm scores at 1-year post-treatment, compared to the HA group, although earlier clinical improvements were superior in the HA group for the initial 3 months.

Level of Evidence: Therapeutic study, Level III.

Keywords: Mesenchymal stem cells, Hyaluronic acid, Osteoarthritis, Knee, Injection

\footnotetext{
* Correspondence: yonggonkoh@gmail.com

Department of Orthopaedic Surgery, Center for Stem Cell \& Arthritis

Research, Yonsei Sarang Hospital, 10, Hyoryeong-ro, Seocho-gu, Seoul,

Republic of Korea
}

(c) The Author(s). 2020 Open Access This article is licensed under a Creative Commons Attribution 4.0 International License, which permits use, sharing, adaptation, distribution and reproduction in any medium or format, as long as you give appropriate credit to the original author(s) and the source, provide a link to the Creative Commons licence, and indicate if changes were made. The images or other third party material in this article are included in the article's Creative Commons licence, unless indicated otherwise in a credit line to the material. If material is not included in the article's Creative Commons licence and your intended use is not permitted by statutory regulation or exceeds the permitted use, you will need to obtain permission directly from the copyright holder. To view a copy of this licence, visit http://creativecommons.org/licenses/by/4.0/. 


\section{Background}

Osteoarthritis (OA) is a prevalent joint disease whose main pathological feature is a chronic cycle of aberrant attempts to repair the implicated joints, thereby leading to inflammation and tissue degradation [37]. The knee is the principal peripheral joint affected, resulting in pain, stiffness, and a progressive loss of function [8]. According to the OA Research Society International, OA management should reduce pain and inflammation, slow down cartilage degradation, improve function, and reduce disability [13]. Treatment can be broadly classified into surgical and non-surgical treatments, and nonsurgical treatments include intra-articular injections, physical modalities, alternative therapies, oral analgesics, and the reduction of modifiable risk factors [2]. Among these, intra-articular injections have been widely used over the past few decades because they present a low risk of complications while providing potential pain relief and improving physical function [57]. Intra-articular injections of hyaluronic acid (HA), a natural glycosaminoglycan found in the synovial fluid which acts as a lubricant and an elastic shock absorber during joint movements [38], can potentially restore the effects of the synovial fluid to protect against cartilage erosion and reduce synovial inflammation [16, 38]. Several studies have demonstrated its beneficial effects, including pain reduction, knee function improvement, and delayed need for arthroplasty [10, 19, 41]. However, the therapeutic effect of HA injections is not permanent [29, 47].

Recently, mesenchymal stem cells (MSCs) have been proposed to be used for cell-based therapies for OA due to their immunomodulatory properties [21, 32]. Considering that the pathogenesis of $\mathrm{OA}$ is based on degeneration and inflammation, the therapeutic properties of MSCs, including paracrine [4, 20], anti-inflammatory [56], and immunomodulatory effects [60], could contribute towards restoring the intra-articular environment [43]. While several studies have reported improvements in clinical outcomes following MSC injection in patients suffering from knee OA [11, 23, 62], only a few studies have compared its clinical outcomes with those of HA injection [35, 55]. Nonetheless, the MSCs used in these studies were derived from the peripheral blood [55], umbilical cord [35], and bone marrow [35]. Therefore, this study aimed to compare the clinical and radiological outcomes of intraarticular injections of adipose-derived MSCs versus HA in patients with knee OA. We hypothesized that patients who received MSC injections would have better outcomes than those treated with HA injection.

\section{Methods}

Patient selection and study design

This study was reviewed and approved by the Institutional Review Board of Yonsei Sarang Hospital (registration number 19-E003-004), and written informed consent was obtained from all participants. We retrospectively reviewed the medical records of 209 consecutive patients with knee OA who were treated with intra-articular injections of MSCs or HA at our clinic and had completed 1 year of follow-up between October 2010 and September 2017. The inclusion criteria were knee OA confirmed by clinical evaluation, radiography, and magnetic resonance imaging (MRI); and symptoms of unilateral knee joint pain and/or functional limitations despite a minimum of 3 months treatment with oral non-steroidal anti-inflammatory drugs. The exclusion criteria were a previous history of HA and/ or steroid injection within 1 year; comorbidities in hip or ankle joints; or hematological or cardiovascular disease(s), systemic infection(s), or immunosuppressive disorder(s). Patients who had knee instability, varus or valgus malalignment of the knee joint of $\geq 5^{\circ}$, metabolic arthritis, joint infections, or large meniscal tears were also excluded. Based on the inclusion and exclusion criteria, 135 patients (MSC injection: 60, HA injection: 75) were eligible. Four of them declined consent and 131 patients were enrolled. After a matching process, 60 patients were finally chosen (Fig. 1). Thirty patients treated with an MSCs were identified and assigned to the MSC group. Thirty patients treated with HA were then matched to the MSC-treated patients, based on gender first, followed by age. The matching process was performed by an independent scientist blinded to the patient's identity, medical history, and clinical complaints, to eliminate potential sources of bias. The minimum followup was 12 months for all patients.

\section{Collection of subcutaneous adipose tissue}

Sample collection and MSC isolation were performed as described previously [30]. Subcutaneous adipose tissue samples were obtained through tumescent liposuction from the gluteal regions of the patients 1 day before MSC injection. We aimed to routinely collect $140 \mathrm{~mL}$ of adipose tissue, of which $120 \mathrm{~mL}$ was used for the injection, and $20 \mathrm{~mL}$ was subjected to laboratory analysis to examine the plastic-adherent cells that form colonyforming unit fibroblast (CFU-F) and confirm the multilineage differentiation of adipose-derived stem cells.

\section{Isolation of stromal vascular fraction and MSCs from subcutaneous adipose tissue}

In the operating room, the aspirated adipose tissue (120 $\mathrm{mL}$ ) was suspended in phosphate-buffered saline solution before being placed in a sterile box and transported to the laboratory. Mature adipocytes and connective tissues were separated from the stromal vascular fraction (SVF) by centrifugation [65]. Prior to insertion, bacteriologic tests were performed to ensure the absence of contamination in the samples, and the viability of cells was assessed using the methylene blue dye exclusion test. 


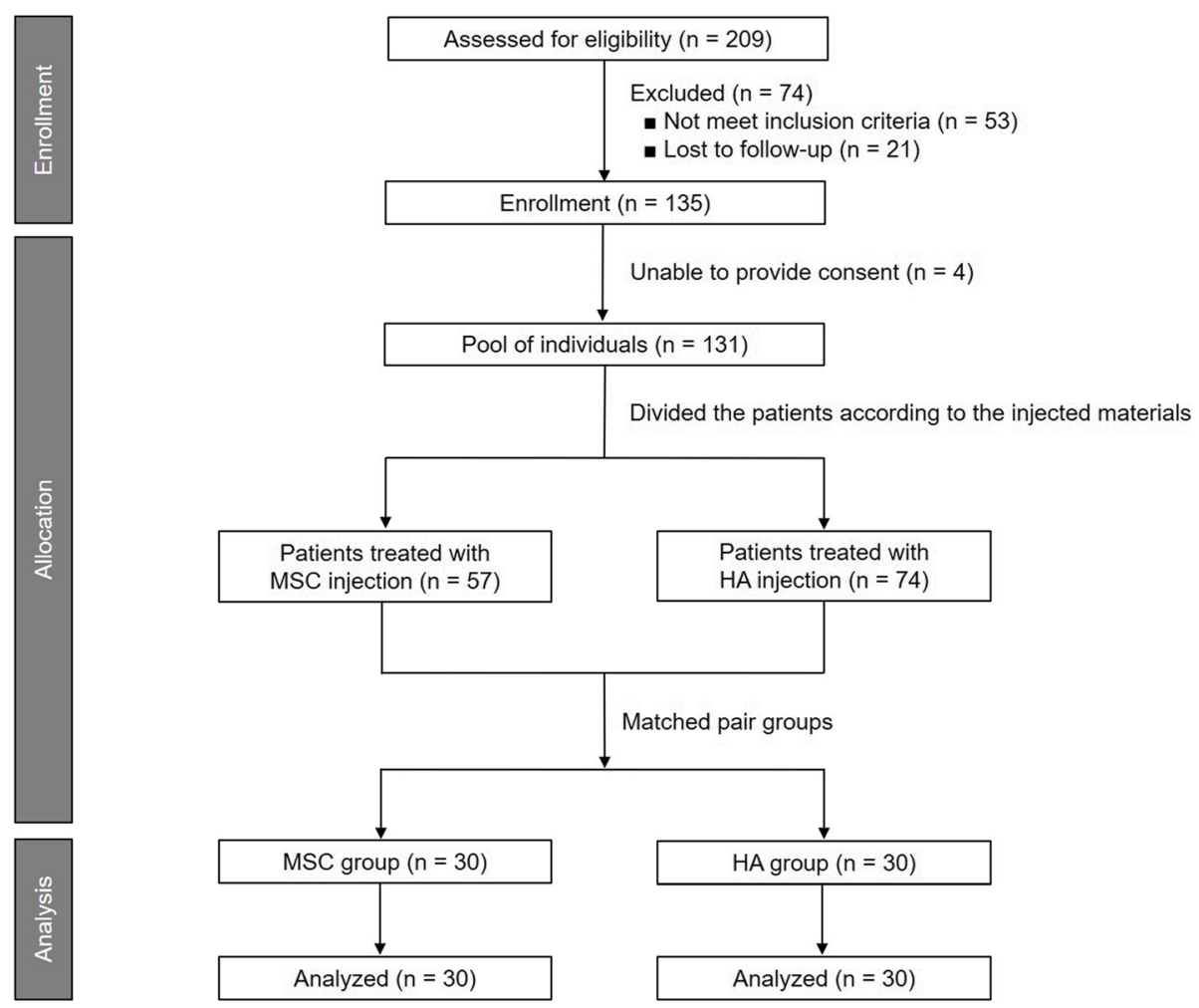

Fig. 1 CONSORT flow diagram depicting the patient enrollment process for this study. MSC, mesenchymal stem cell; HA, hyaluronic acid

The remaining $20 \mathrm{~mL}$ of adipose tissue was processed by the same method and used for cell analysis.

\section{Assessment of plastic-adherent cells that form CFU-F and immunophenotyping of adipose-derived stem cells}

MSCs were originally referred to as fibroblastoid colonyforming-cells because one of their characteristic features is adherence to tissue culture plastic and generation of colonies when plated at low densities $[14,45]$. The CFU-F assay were examined to confirm the ability to generate mesenchymal progenitors of the adipose-derived stem cells. The cells were cultured in T25 flasks at a final concentration of 16 cells $/ \mathrm{cm}^{2}$ to evaluate the frequency of mesenchymal-like progenitors. Colonies of $\geq 50$-cell aggregates were scored under an optical microscope to assess the ability to form colonies. Cells regularly seeded at 50 cells $/ \mathrm{cm}^{2}$ were expanded till reaching the adequate number for analyzing the flow cytometric immunophenotype using fluorescence-activated cell sorting (FACS). MSC marker phenotyping was performed using CD14, CD34, CD90, and CD105 antibodies according to an established protocol $[9,49]$. For analysis of the flow cytometric immunophenotype using FACS, $2 \times 10^{6}$ of cells are required per a CD marker. Therefore, we obtained $8 \times 10^{6}$ of cells for four CD markers by culture expansion process.
Confirmation of multilineage differentiation of adiposederived stem cells

Adipose-derived stem cells were plated at $5 \times 10^{3}$ cells/ $\mathrm{cm}^{2}$ in Dulbecco's modified Eagle's medium (Hyclone, Logan, UT) supplemented with $10 \%$ fetal bovine serum (Hyclone, Logan, UT), and allowed to adhere for $24 \mathrm{~h}$. The culture medium was then replaced with specific inductive media in order to determine the adipogenic, osteogenic, and chondrogenic differentiation potential [34]. We evaluated the capacity of the human subcutaneous adipose tissue to generate mesenchymal progenitors according to CFU-F.

\section{Injection of mesenchymal stem cells or hyaluronic acid}

All injections in both groups were performed using the same technique by an experienced senior orthopedic surgeon. Patients lay down on the table in a supine position with their knees extended during intra-articular injection. An arthrocentesis was performed to eliminate a knee effusion, before MSC or HA were administered by transversely inserting a needle between the articular surface and patellofemoral joint in the midpoint of the patella, after pushing the patella upwards and shifting it to the lateral side [52]. $3.0 \mathrm{~mL}$ of sodium hyaluronate [20 mg/mL, molecular weight: $\left.6 \times 10^{6} \mathrm{Da}(\mathrm{Da})\right]$ was used for HA injection. The patients were advised against additional 
treatments including physical therapy, acupuncture, steroid injection, and opioid or strong analgesics until 1 year after the injection. They were also told to avoid weight-bearing motions that impose an excessive burden on the affected knee, such as standing for prolonged periods, jogging, and lifting heavy objects, for the first 3 days.

\section{Outcome assessments}

All patients were evaluated clinically and radiologically before injection and during follow-up(s). For clinical evaluation, the visual analog scale (VAS), International Knee Documentation Committee (IKDC) [22], and Lysholm [31] scores were collected. Adverse events were recorded for safety evaluation. Radiological evaluations included a weight-bearing anterior-posterior (AP) view, true lateral view at $30^{\circ}$ of knee flexion, and hip-to-ankle standing AP radiograph on a long cassette. To avoid potential bias, an independent observer, who was a musculoskeletal-trained radiologist not involved in the care of patients and blinded to the intention of this study, performed the radiological evaluation. The Kellgren-Lawrence (K-L) grading system [25] was used to assess the AP radiographs.

\section{Statistical analysis}

The matching process was performed based on gender and age using nearest neighbor matching. To determine the necessary power for our study, an a priori power analysis based on IKDC was performed. Based on our a priori power analysis to obtain a power of 0.80 or higher with a ratio of 1:1, we need a minimum of 30 patients in each group. The primary dependent variables were VAS, IKDC, and Lysholm scores as clinical outcomes, and K-L grade as radiological outcome. Descriptive statistics were calculated as mean \pm standard deviation for continuous variables and frequencies and proportions for categorical variables. The Wilcoxon signed-rank test was performed to compare the pre- and post-operative clinical values over the follow-up period, while the Mann-Whitney $U$ test was used to compare the results between the two groups. The Fisher exact test was used to compare the categorical data. Statistical analyses were performed using SPSS (v13.0; IBM Corp., Armonk, NY, USA), with a $P$ value $<0.05$ being considered as statistically significant.

\section{Results}

\section{General characteristics}

The final study population included 22 men and 38 women with a mean age of 63.1 years (range: 56-71 years). There was no significant difference for body mass index, side of involvement, follow-up period, and K-L grade between the groups (Table 1). No clinically significant adverse event was noted during the 1-year followup period. Although mild swelling of knee joints was observed in three cases (MSC group: 2, HA group: 1), it was resolved without intervention. Subcutaneous induration was observed at the fat harvest site at the buttock area in three cases in the MSC group; however, it was also resolved without intervention by the 6-months follow-up.

\section{Isolation and characterization of cells}

The isolation and characterization procedures determined that adipose-derived stem cells made up 9.4\% (range: $8.5-11.3 \%$ ) of the SVF. Consequently, an average of $7.6 \times 10^{7}$ cells in the SVF, which contained an average of $7.1 \times 10^{6}$ stem cells (range: $6.5 \times 10^{6}-8.6 \times 10^{6}$ cells), were used for MSC injection. FACS characterization indicated positive expressions of CD90 (99.17\%) and CD105 (94.62\%), and negative expressions of CD34 (5.34\%) and CD14 (2.64\%). The treated stem cells exhibited adipogenic, osteogenic, and chondrogenic differentiation potentials after staining.

Table 1 Comparison of baseline demographics in the study groups

\begin{tabular}{llll}
\hline & MSC $(\boldsymbol{n}=\mathbf{3 0})$ & HA $(\boldsymbol{n}=\mathbf{3 0})$ & $\boldsymbol{P}$ value \\
\hline Age, years & $63.0 \pm 3.2(57-70)$ & $63.2 \pm 3.8(56-71)$ & 0.769 \\
Gender, male/female, $\mathrm{n}$ & $11 / 19$ & $11 / 19$ & 0.999 \\
Body mass index, $\mathrm{kg} / \mathrm{m}^{2}$ & $26.4 \pm 1.5(22.9-28.9)$ & $26.6 \pm 1.5(24.5-29.5)$ & 0.546 \\
Side of involvement, right/left, $\mathrm{n}$ & $14 / 16$ & $18 / 12$ & 0.309 \\
Follow-up period, months & $14.2 \pm 3.4(12-24)$ & $15.2 \pm 3.9(12-25)$ & 0.293 \\
Kellgren-Lawrence grade, $\mathrm{n}(\%)$ & & $3(10.0)$ & 0.769 \\
1 & $2(6.7)$ & $9(30.0)$ & $13(43.3)$ \\
2 & $10(33.3)$ & $5(16.7)$ \\
\hline
\end{tabular}

Data are expressed as mean \pm standard deviation (range) unless otherwise indicated. MSC Mesenchymal stem cell, HA Hyaluronic acid 


\section{Clinical outcomes}

The mean VAS, IKDC, and Lysholm scores at baseline and at 1-month, 3-months, 6-months, and 1-year followups are summarized in Table 2. The mean VAS score in the MSC group significantly and progressively decreased until 1-year post-treatment as compared to baseline $(P<0.05$ for all; Fig. 2a). On the contrary, the mean VAS score in the HA group significantly decreased at 1 month post-injection with reference to the baseline $(P<0.05)$, slightly increased at the 3 -month follow-up $(P=0.317)$, and gradually increased significantly from 3 months to 1 -year post-injection $(P<0.05)$ (Fig. 2a). A significantly greater pain relief was reported in the MSC group compared to that in the HA group at the 1-year follow-up ( $P=0.041$; Table 2). The mean IKDC and Lyshlom scores in both groups showed similar trends over the follow-up period. In the MSC group, the mean IKDC and Lysholm scores were significantly improved at 1 month after injection as compared to the baselines $(P<0.001$ for both). Further significant improvements in the mean IKDC and Lysholm scores were observed until 1-year post-treatment $(P<0.05$ for all; Fig. $2 \mathrm{~b}$ and c). In the HA group, the mean IKDC and Lysholm scores were also significantly improved at 1-month and 3 -months post-injection as compared to the baseline $(P<0.001$ for all; Fig. $2 \mathrm{~b}$ and $\mathrm{c})$. However, the mean IKDC and Lysholm scores gradually decreased from 3-

Table 2 Comparison of preoperative and postoperative clinical outcomes

\begin{tabular}{llll}
\hline & MSC & HA & $P$ value \\
\hline VAS & & & \\
Baseline & $8.4 \pm 1.1$ & $8.1 \pm 1.1$ & 0.346 \\
1 month & $5.4 \pm 1.4$ & $4.7 \pm 1.0$ & 0.039 \\
3 months & $5.2 \pm 1.3$ & $4.7 \pm 1.1$ & 0.111 \\
6 months & $5.0 \pm 1.2$ & $5.1 \pm 0.9$ & 0.809 \\
1 year & $4.8 \pm 1.1$ & $5.4 \pm 1.0$ & 0.041 \\
IKDC score & & & \\
Baseline & $37.1 \pm 7.8$ & $39.2 \pm 6.3$ & 0.256 \\
1 month & $55.5 \pm 8.2$ & $62.7 \pm 8.0$ & 0.001 \\
3 months & $61.1 \pm 8.0$ & $65.5 \pm 6.6$ & 0.025 \\
6 months & $64.6 \pm 6.1$ & $64.9 \pm 7.2$ & 0.893 \\
1 year & $66.0 \pm 5.2$ & $62.0 \pm 6.9$ & 0.014 \\
Lysholm score & & & \\
Baseline & $54.4 \pm 6.3$ & $55.2 \pm 5.7$ & 0.640 \\
1 month & $68.6 \pm 6.6$ & $72.8 \pm 7.5$ & 0.024 \\
3 months & $72.7 \pm 7.1$ & $74.8 \pm 7.5$ & 0.260 \\
6 months & $76.7 \pm 6.8$ & $74.6 \pm 5.7$ & 0.199 \\
1 year & $77.6 \pm 6.3$ & $73.9 \pm 5.9$ & 0.020 \\
\hline Data expred as & & & \\
\hline
\end{tabular}

Data are expressed as mean \pm standard deviation. MSC Mesenchymal stem cell HA Hyaluronic acid, VAS Visual analog scale, IKDC International Knee Documentation Committee months to 1-year post-injection, albeit no significant difference $(P>0.05$ for all; Fig. $2 b$ and $c)$ with the exception of the IKDC score at 1-year post-injection with reference to that at 6 -months post-injection $(P=0.001$; Fig. $2 \mathrm{~b})$. There were significant differences in the IKDC scores at 1-month, 6-months, and 1-year, and in the Lysholm scores at 1-month and 1-year post-injection between the groups (Table 2). Although more pronounced clinical improvements were evident in the HA group during the early follow-up periods, the MSC group eventually exhibited superior clinical improvements at the 1-year follow-up.

\section{Radiological outcomes}

According to the K-L grading system, a majority of the affected knees were categorized as grade 2 or 3 before injection (73.3\% in both groups; Table 1), and there was no significant difference in $\mathrm{K}-\mathrm{L}$ grade between the groups $(P=0.769$; Table 1$)$. The $\mathrm{K}-\mathrm{L}$ grade worsened in a few patients (Figs. 3 and 4 ) especially in the HA group (6.67\% in MSC group and $20 \%$ in HA group), and the majority of the knees were categorized as grade 3 or 4 at 1-year post-treatment (MSC group: 63.3\%, HA group: $70.0 \%)$. However, there was no significant difference in $\mathrm{K}-\mathrm{L}$ grade between the groups at 1-year post-injection $(P=0.742)$. We compared the preoperative and postoperative clinical outcomes according to the presence of radiological worsening in both groups, and found no significant differences (Table 3).

\section{Discussion}

To date, no studies have compared between adiposederived MSC and HA. The identification of changes in clinical outcomes following MSC injection and the comparison to those observed following HA injection would provide patients with more accurate expectations of this treatment. To our knowledge, this is the first matchedpair study comparing the outcomes of an intra-articular injection of adipose-derived MSCs versus HA for the treatment of knee OA. Principally, both MSCs and HA resulted in clinical improvements. However, improvements were only continual and significant in the MSC group throughout the follow-up period. While the clinical outcomes were significantly enhanced and superior in the HA group until the 3-months follow-up, the improved conditions gradually reverted thereafter. Ultimately, MSC treatment surpassed HA treatment in begetting better clinical outcomes at the 1-year followup. On the other hand, the radiological outcomes of most patients remained unchanged in terms of $\mathrm{K}-\mathrm{L}$ grading. Nevertheless, there were more patients in the HA group that showed signs of deterioration with K-L grade progression as compared to those in the MSC group, albeit no statistical significance. 

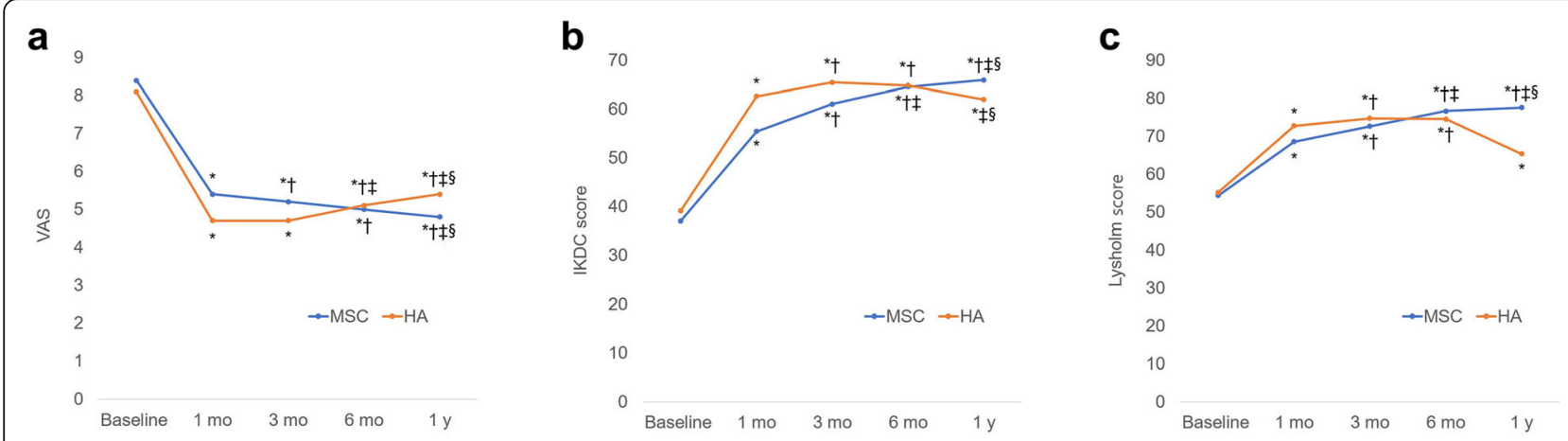

Fig. 2 Graphs demonstrating the changes in the a visual analog scale (VAS), $\mathbf{b}$ International Knee Documentation Committee (IKDC), and c Lysholm scores. Significantly different from ${ }^{*}$ baseline, ${ }^{\dagger} 1$-month follow-up, ${ }^{\ddagger} 3$-month follow-up, ${ }^{\S} 6$-month follow-up

Although the exact mechanism of HA in knee OA has not been elucidated, its clinical effects are probably mediated by several factors. Clinical studies revealed that pain relief may be attributed to the effects of HA on nerve impulses and sensitivities; HA reduces the activity of pain-related primary afferents by coating the pain receptors in the synovial tissues, and perhaps also traps molecules involved in pain signaling [44]. Several in vitro studies also indicated that HA administration can enhance the synthesis of extracellular matrix (ECM) proteins, which is helpful for restoring the cartilage matrix in diseased articular cartilage in OA-affected knee joints $[24,26,48,50]$. Furthermore, in vivo studies $[1,39]$ demonstrated the ability of HA to prevent the release of ECM proteins from the cartilage matrix into joint space. Some studies also suggested that HA can suppress the production and activity of proinflammatory mediators and proteases, and alter the function of certain immune cells $[15,54]$. Histological evidence showed that HA prevents cartilage degradation and may even promote its regeneration [17]. Collectively, HA seems to function mainly by reducing pain transmission and blunting inflammatory cascade. and stimulating synthesis and deposition of ECM molecules which are suppressed in implicated OA joints [40]. Similar to HA, MSCs have been reported to alleviate OA joint degeneration by improving the local microenvironment, immune-regulation, and anti-inflammatory biological activities through the secretion of exosomes, growth factors, cytokines, anti-inflammatory factors, and other bioactive molecules [59]. MSCs have also been shown to promote the proliferation of a pool of endogenous cells and contribute to

\section{a}

\section{K-L grade \\ (baseline)}

Grade 1

2

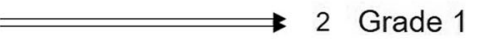

Grade 2

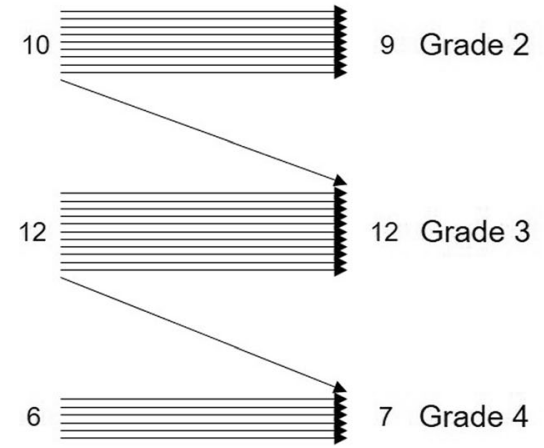

Grade 3

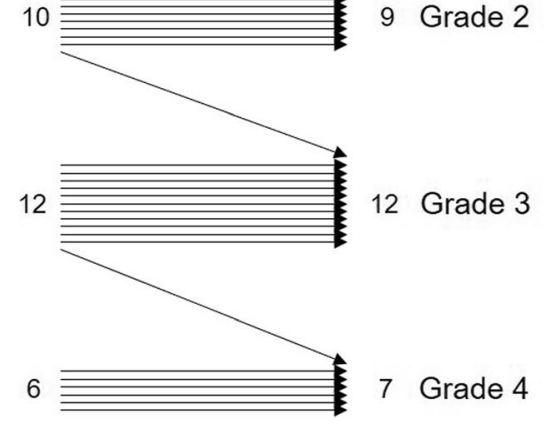

Grade 4
K-L grade

(1y after injection)

2 Grade 1

12 Grade 3

b

K-L grade

(baseline)

K-L grade

(1y after injection)

Fig. 3 Changes in Kellgren-Lawrence (K-L) grades from baseline to 1-year post-injection in MSC $\mathbf{a}$ and HA $\mathbf{b}$ groups 


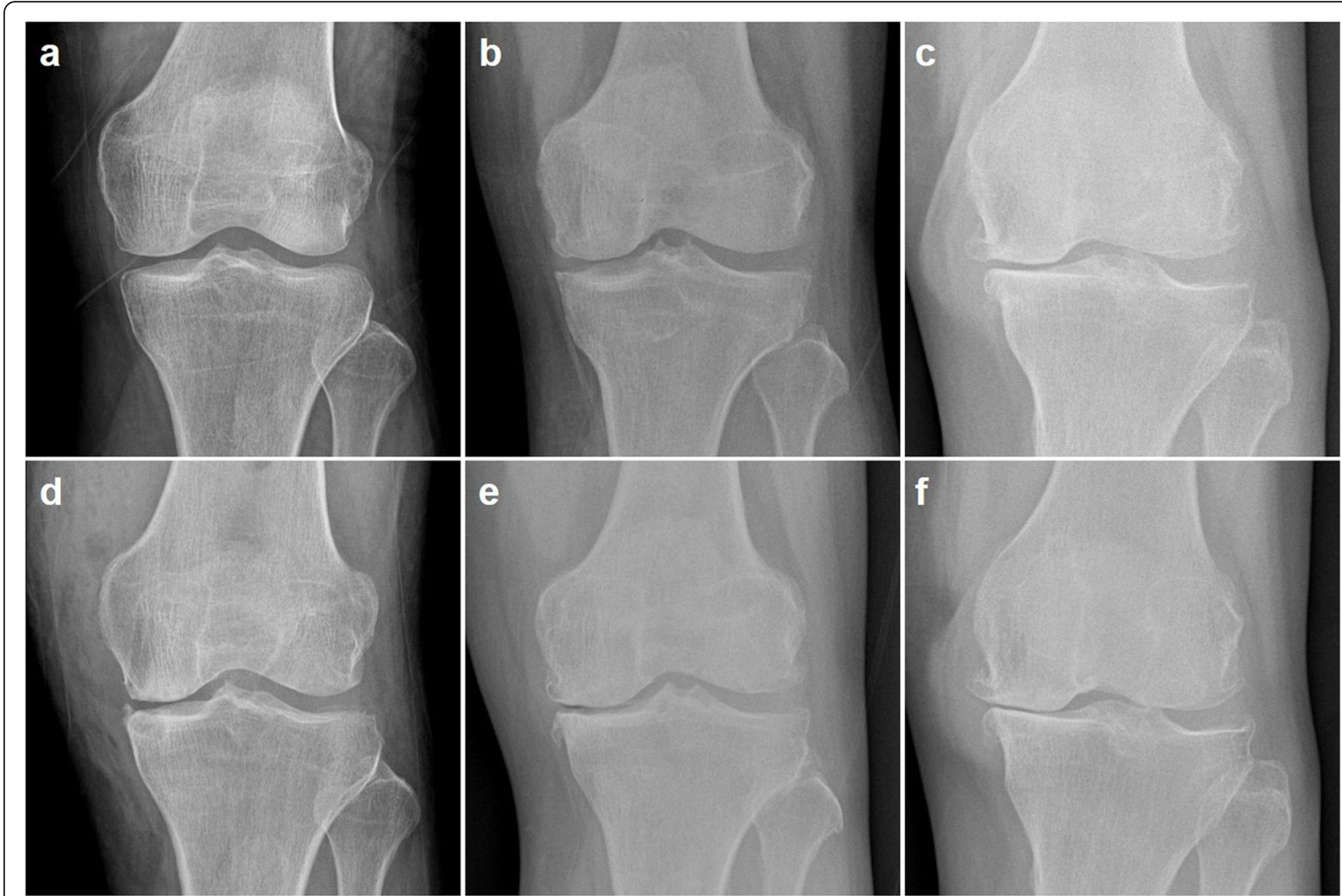

Fig. 4 Radiographs showing the weight-bearing anterior-posterior (AP) views at baseline (a-c) and 1 year after injection (d-f). Radiographs of a 59-year-old man revealing a progression of knee osteoarthritis (OA) from Kellgren-Lawrence (K-L) grade 1 at baseline (a) to K-L grade 2 at 1 year after hyaluronic acid (HA) injection (d). Radiographs of a 64-year-old woman demonstrating an advancement of knee OA from K-L grade 2 at baseline (b) to K-L grade 3 at 1 year after mesenchymal stem cell (MSC) injection (e). Radiographs of a 62-year-old woman depicting a deterioration of knee OA from K-L grade 3 at baseline (c) deteriorating to K-L grade 4 at 1 year after HA injection (f)

chondrogenesis by renewing the ECM and synthesizing type II collagen [64]. Despite the capability of MSCs to differentiate into mesodermal cell lineages including cartilage, initial regenerative claims concerning therapeutic effects in $\mathrm{OA}$ have been revised due to recent evidence suggesting that paracrine and anti-inflammatory actions are crucial for tissue-restoring effects of MSC treatments [58]. Therefore, we investigated and compared the clinical outcomes over the 1-year follow-up period after intra-articular injections of either MSCs or HA. We speculate that the short-term clinical outcomes were mainly affected by the enhancement of the microenvironment, immune-regulation and anti-inflammatory effects rather than cartilage regeneration itself, because cartilage regeneration would probably require a longer time to occur or would not happen even at longer follow up after MSC injections as described in several previous studies $[18,33]$.

The efficacy of HA injections on pain relief and joint function restoration has been evaluated in numerous studies $[10,19,26,40,41,51]$, and recent systematic reviews have shown that improvements in pain intensity and functional outcomes were likely to take effect in 48 weeks and could last up to 6 months $[3,47]$. Moreover, Suppan et al. [51] suggested that HA efficacy was sustainable for $\geq 1$ year. Our findings were consistent with previously reported action duration of intra-articular injections of HA for knee OA. While the clinical outcomes in the HA group significantly improved only until 3 months and gradually reduced thereafter (Table 2 and Fig. 2), the post-treatment scores were still significantly better than the baseline scores; this implied that HA efficacy lasted for $\geq 1$ year. Interestingly, a greater improvement in clinical outcomes was noted in the HA group in comparison to the MSC group for the first 3 months (Table 2). We speculate that these results were due to the antinociceptive effect of HA. Boettger et al. [7] reported that a single intra-articular injection of HA could alleviate pain by more than $50 \%$ in comparison to saline in a bradykinin/prostaglandin E2 animal model and the pain responses lasted for $\geq 56$ days after administration. In addition, HA exposure has been shown to decrease arachidonic acid secretion from the fibroblasts isolated 
Table 3 Comparison of preoperative and postoperative clinical outcomes according to the presence of radiological worsening in both groups

\begin{tabular}{|c|c|c|c|c|c|c|}
\hline \multirow[t]{3}{*}{ 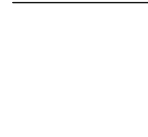 } & \multicolumn{3}{|l|}{ MSC } & \multicolumn{3}{|l|}{ HA } \\
\hline & \multicolumn{3}{|c|}{ Radiological worsening } & \multicolumn{3}{|c|}{ Radiological worsening } \\
\hline & Presence $(n=2)$ & Absence $(n=28)$ & $P$ value $^{\mathrm{a}}$ & Presence $(n=6)$ & Absence $(n=24)$ & $P$ value ${ }^{\mathrm{a}}$ \\
\hline \multicolumn{7}{|l|}{ VAS } \\
\hline Baseline & $8.0 \pm 1.4$ & $8.4 \pm 1.1$ & 0.604 & $8.5 \pm 1.0$ & $8.0 \pm 1.1$ & 0.359 \\
\hline 1 year & $5.5 \pm 2.1$ & $4.8 \pm 1.1$ & 0.372 & $5.7 \pm 1.0$ & $5.3 \pm 1.0$ & 0.404 \\
\hline$P$ value $^{\mathrm{b}}$ & $<0.001$ & $<0.001$ & & $<0.001$ & $<0.001$ & \\
\hline \multicolumn{7}{|l|}{ IKDC score } \\
\hline Baseline & $42.0 \pm 11.3$ & $36.7 \pm 7.7$ & 0.366 & $35.5 \pm 4.6$ & $40.1 \pm 6.4$ & 0.110 \\
\hline 1 year & $68.0 \pm 8.5$ & $65.9 \pm 5.1$ & 0.588 & $60.3 \pm 6.1$ & $62.5 \pm 7.2$ & 0.512 \\
\hline$P$ value ${ }^{b}$ & $<0.001$ & $<0.001$ & & $<0.001$ & $<0.001$ & \\
\hline \multicolumn{7}{|c|}{ Lysholm score } \\
\hline Baseline & $57.5 \pm 10.6$ & $54.2 \pm 6.2$ & 0.489 & $51.7 \pm 4.2$ & $56.0 \pm 7.8$ & 0.095 \\
\hline 1 year & $78.0 \pm 11.3$ & $77.6 \pm 6.1$ & 0.927 & $70.8 \pm 5.8$ & $74.6 \pm 5.7$ & 0.160 \\
\hline$P$ value ${ }^{b}$ & $<0.001$ & $<0.001$ & & $<0.001$ & $<0.001$ & \\
\hline
\end{tabular}

Data are expressed as mean \pm standard deviation. MSC Mesenchymal stem cell, HA Hyaluronic acid, VAS Visual analog scale, IKDC International Knee Documentation Committee

${ }^{a}$ Mann-Whitney U test

${ }^{b}$ Wilcoxon signed-rank test for comparison of clinical outcomes at baseline versus I year after the injection

from patients with knee OA under bradykinin stimulation or calcium ionophore induction [53], which contributes to its antinociceptive effects. Furthermore, a recent in vitro study demonstrated that the stimulation of $\mathrm{K}$ opioid receptors by HA [63]. Therefore, the superior clinical outcomes in the HA group for the first threemonth scan be explained by these antinociceptive effects of HA. A previous meta-analysis suggested that intraarticular MSC injections could significantly improve pain scores for knee OA, which indicated that MSCs can be used for pain relief in short-term follow-up [27]. The analgesic effect of MSCs was also demonstrated by Orozco et al. [42] They evaluated the pain, disability, and quality of life after an intra-articular injection of bone marrowderived MSCs in twelve patients with knee OA and found that the patients exhibited rapid and progressive improvement in algofunctional indices of up to $65-78 \%$ by 1 year post-treatment. They also reported a rather quick onset of analgesic effect, with more than $50 \%$ of the maximum improvement attained by the third month., but a tapered effect subsequently to reach the maximum effect at the 1-year follow-up. Similar findings were observed in our study; the mean VAS score in the MSC group gradually improved to 4.8 points at the 1year follow-up, which was similar with the mean VAS score in the HA group at the 1-month follow-up (4.7 points). It should be noted that the improved clinical outcomes in the HA group deteriorated at some point during the follow-up period, while a steady improvement in clinical outcomes was achieved in the MSC group. Moreover, a greater improvement was eventually achieved at the 1-year follow-up in the MSC group than in the HA group (Table 2). Taken together, we consider that MSCs has a superior longevity to HA for its therapeutic effects especially in pain relief. In addition to the analgesic effects, a systemic review and meta-analysis proposed that MSC injection could be potentially efficacious not only for decreasing pain but also for improving physical function in patients with knee OA [61]. Another meta-analysis also demonstrated that the use of a recommended concentration of MSCs may result in favorable clinical outcomes; however, current evidence does not support the use of intra-articular injections of MSCs for cartilage repair in knee OA [28]. Here, we focused on investigating the clinical outcomes without evaluating the cartilage repair status due to a lack of evaluation tools. Given that no similar studies have been published, we believe that our findings are valuable in comparing the outcomes of MSCs versus HA treatments for knee OA.

Literature reviews revealed that the progression of knee OA is natural and inevitable [12, 36]. Several studies estimated the narrowing of joint space in patients suffering from knee $\mathrm{OA}$ to be $0.1-0.3 \mathrm{~mm} /$ year $[6,36]$. A study involving 869 patients with knee OA revealed $\mathrm{K}-\mathrm{L}$ grade worsening in $3 \%$ males and $4 \%$ females each year [12]. Herein, the K-L grade exacerbated in a few patients at 1-year post-injection, especially those in the HA group (Figs. 3 and 4). Although no statistically significant difference was found in the K-L grades between the two treatment groups at the 1-year follow-up $(P=$ 0.742 ), we hypothesize that MSCs could possibly retard 
the progression of arthritic changes to a larger extent as compared to HA. However, the follow-up period of our study was relatively short (mean; MSC group: 14.2 months, HA group: 15.2 months). Therefore, long-term evaluations are required to investigate the effects of MSCs or HA on the progression of knee OA.

There are several limitations in the present study. First, we were unable to conduct this study in a blinded or randomized manner, and the data were collected retrospectively. However, case-matching according to gender and age in combination with strict inclusion and exclusion criteria were set to achieve a relatively homogeneous distribution of all the parameters that can potentially influence the post-treatment clinical outcomes. Therefore, we believe that these data are valuable in comparing the outcomes of MSCs versus HA in treating knee OA. Second, only VAS, IKDC, and Lysholm scores were considered for clinical evaluation and K-L grade was assessed for radiological evaluation. Other more specific OA-related scores such as Knee Injury and Osteoarthritis Outcome Score [46] or the Western Ontario and McMaster University Osteoarthritis Index [5] would have been more suitable. In addition, it is also important to examine the morphological changes of the articular cartilage for a more precise therapeutic evaluation. Hence, follow-up MRI or diagnostic and/or therapeutic arthroscopy is needed to analyze the association of MSC or HA injection and the mechanical properties and biological functions of the regenerative cartilage. Third, the optimal number of MSCs and dosage of HA remain unknown. Presently, low $\left(0.5 \times 10^{6}-1 \times 10^{6} \mathrm{Da}\right)$, intermediate $\left(2 \times 10^{6} \mathrm{Da}\right)$, or high $\left(6 \times 10^{6} \mathrm{Da}\right)$ molecular weights (MW) of HA are marketed; it is recommended to administer the low and intermediate MW of HA weekly for three to five doses, and the high MW of HA as a single larger dose [51]. In this study, high MW of HA was used. However, further investigation is required to study the volume effect of single larger dose versus multiple smaller doses of intra-articular injection of high MW of HA in achieving maximum therapeutic effects in knee OA. Furthermore, studies are required to determine the optimal number of MSCs to be used for better clinical outcomes in the treatment of knee OA. In addition, an arthrocentesis was performed to eliminate a knee effusion before MSC or HA were administered. However, the effusion, especially requiring arthrocentesis, can deeply affect the injection results. The future study investigating the effect of effusion on the injection results is required for more precise comparing the outcomes of MSCs versus HA.

\section{Conclusion}

This study showed that intra-articular injections of MSCs and HA improved the clinical outcomes of patients suffering from knee OA, without severe adverse effects, throughout the short-term follow-up period. Although more pronounced clinical improvements were evident in the HA group during the early follow-up periods, the MSC group eventually exhibited superior clinical improvements at the 1-year follow-up. However, a larger sample size and long-term prospective randomized studies are needed to validate our findings.

\section{Acknowledgments}

All authors declare no funding sources or sponsors in this study. The authors would like to thank the staff and participants in the Center for Stem Cell and Arthritis Research of Yonsei Sarang Hospital.

\section{Authors' contributions}

YSK, YBK, TYK and YGK carried out the design of the study, participated in collection and analysis of data, and drafted the manuscript. YSK, DSS, DHT and PKC conceived of the study, and participated in its design and coordination and helped to draft the manuscript. YSK and YGK participated in analysis and interpretation of data and critical revision of the manuscript. All authors read and approved the final manuscript.

\section{Funding}

No funding

\section{Availability of data and materials}

The datasets used and/or analysed during the current study are available from the corresponding author on reasonable request.

\section{Competing interests}

The authors declare that they have no competing interests.

Received: 28 September 2020 Accepted: 9 November 2020 Published online: 13 November 2020

\section{References}

1. Abatangelo G, Botti P, Del Bue M, Gei G, Samson JC, Cortivo R, De Galateo A, Martelli M (1989) Intraarticular sodium hyaluronate injections in the pond-Nuki experimental model of osteoarthritis in dogs. I. Biochemical results. Clin Orthop Relat Res 241:278-285

2. Abramoff B, Caldera FE (2020) Osteoarthritis: pathology, diagnosis, and treatment options. Med Clin North Am 104(2):293-311

3. Bannuru RR, Natov NS, Dasi UR, Schmid CH, McAlindon TE (2011) Therapeutic trajectory following intra-articular hyaluronic acid injection in knee osteoarthritis--meta-analysis. Osteoarthr Cartil 19(6):611-619

4. Barry F, Murphy M (2013) Mesenchymal stem cells in joint disease and repair. Nat Rev Rheumatol 9(10):584-594

5. Bellamy N, Buchanan WW, Goldsmith CH, Campbell J, Stitt LW (1988) Validation study of WOMAC: a health status instrument for measuring clinically important patient relevant outcomes to antirheumatic drug therapy in patients with osteoarthritis of the hip or knee. J Rheumatol 15(12):1833-1840

6. Bingham CO 3rd, Buckland-Wright JC, Garnero P, Cohen SB, Dougados M, Adami S, Clauw DJ, Spector TD, Pelletier JP, Raynauld JP, Strand V, Simon LS, Meyer JM, Cline GA, Beary JF (2006) Risedronate decreases biochemical markers of cartilage degradation but does not decrease symptoms or slow radiographic progression in patients with medial compartment osteoarthritis of the knee: results of the two-year multinational knee osteoarthritis structural arthritis study. Arthritis Rheum 54(11):3494-3507

7. Boettger MK, Kummel D, Harrison A, Schaible HG (2011) Evaluation of long-term antinociceptive properties of stabilized hyaluronic acid preparation (NASHA) in an animal model of repetitive joint pain. Arthritis Res Ther 13(4):R110

8. Cross M, Smith E, Hoy D, Nolte S, Ackerman I, Fransen M, Bridgett L, Williams S, Guillemin F, Hill CL, Laslett LL, Jones G, Cicuttini F, Osborne R, Vos T, Buchbinder R, Woolf A, March L (2014) The global burden of hip and knee osteoarthritis: estimates from the global burden of disease 2010 study. Ann Rheum Dis 73(7):1323-1330 
9. de Girolamo L, Niada S, Arrigoni E, Di Giancamillo A, Domeneghini C, Dadsetan M, Yaszemski MJ, Gastaldi D, Vena P, Taffetani M, Zerbi A, Sansone V, Peretti GM, Brini AT (2015) Repair of osteochondral defects in the minipig model by OPF hydrogel loaded with adipose-derived mesenchymal stem cells. Regen Med 10(2):135-151

10. DeCaria JE, Montero-Odasso M, Wolfe D, Chesworth BM, Petrella RJ (2012) The effect of intra-articular hyaluronic acid treatment on gait velocity in older knee osteoarthritis patients: a randomized, controlled study. Arch Gerontol Geriatr 55(2):310-315

11. Doyle EC, Wragg NM, Wilson SL (2020) Intraarticular injection of bone marrow-derived mesenchymal stem cells enhances regeneration in knee osteoarthritis. Knee Surg Sports Traumatol Arthrosc. PMID: 32006075. https:// doi.org/10.1007/s00167-020-05859-z.

12. Felson DT, Zhang Y, Hannan MT, Naimark A, Weissman BN, Aliabadi P, Levy D (1995) The incidence and natural history of knee osteoarthritis in the elderly. The Framingham osteoarthritis study. Arthritis Rheum 38(10):1500-1505

13. Fibel KH, Hillstrom HJ, Halpern BC (2015) State-of-the-art management of knee osteoarthritis. World J Clin Cases 3(2):89-101

14. Friedenstein AJ, Chailakhyan RK, Latsinik NV, Panasyuk AF, Keiliss-Borok IV (1974) Stromal cells responsible for transferring the microenvironment of the hemopoietic tissues. Cloning in vitro and retransplantation in vivo. Transplantation 17(4):331-340

15. Fukuda K, Oh M, Asada S, Hara F, Matsukawa M, Otani K, Hamanishi C (2001) Sodium hyaluronate inhibits interleukin-1-evoked reactive oxygen species of bovine articular chondrocytes. Osteoarthr Cartil 9(4):390-392

16. Gomis A, Miralles A, Schmidt RF, Belmonte C (2009) Intra-articular injections of hyaluronan solutions of different elastoviscosity reduce nociceptive nerve activity in a model of osteoarthritic knee joint of the Guinea pig. Osteoarthr Cartil 17(6):798-804

17. Guidolin DD, Ronchetti IP, Lini E, Guerra D, Frizziero L (2001) Morphological analysis of articular cartilage biopsies from a randomized, clinical study comparing the effects of 500-730 kDa sodium hyaluronate (Hyalgan) and methylprednisolone acetate on primary osteoarthritis of the knee. Osteoarthr Cartil 9(4):371-381

18. Gupta PK, Chullikana A, Rengasamy M, Shetty N, Pandey V, Agarwal V, Wagh SY, Vellotare PK, Damodaran D, Viswanathan P, Thej C, Balasubramanian S, Majumdar AS (2016) Efficacy and safety of adult human bone marrowderived, cultured, pooled, allogeneic mesenchymal stromal cells (Stempeucel ${ }^{\circledast}$ ): preclinical and clinical trial in osteoarthritis of the knee joint. Arthritis Res Ther 18(1):301

19. He WW, Kuang MJ, Zhao J, Sun L, Lu B, Wang Y, Ma JX, Ma XL (2017) Efficacy and safety of intraarticular hyaluronic acid and corticosteroid for knee osteoarthritis: a meta-analysis. Int J Surg 39:95-103

20. Horie M, Choi H, Lee RH, Reger RL, Ylostalo J, Muneta T, Sekiya I, Prockop DJ (2012) Intra-articular injection of human mesenchymal stem cells (MSCs) promote rat meniscal regeneration by being activated to express Indian hedgehog that enhances expression of type II collagen. Osteoarthr Cartil 20(10):1197-1207

21. Im Gl (2018) Tissue engineering in osteoarthritis: current status and Prospect of Mesenchymal stem cell therapy. BioDrugs 32(3):183-192

22. Irrgang JJ, Anderson AF, Boland AL, Harner CD, Kurosaka M, Neyret $P$, Richmond JC, Shelborne KD (2001) Development and validation of the international knee documentation committee subjective knee form. Am J Sports Med 29(5):600-613

23. Jo CH, Chai JW, Jeong EC, Oh S, Shin JS, Shim H, Yoon KS (2017) Intra-articular injection of Mesenchymal stem cells for the treatment of osteoarthritis of the knee: a 2-year follow-up study. Am J Sports Med 45(12):2774-2783

24. Kawasaki K, Ochi M, Uchio Y, Adachi N, Matsusaki M (1999) Hyaluronic acid enhances proliferation and chondroitin sulfate synthesis in cultured chondrocytes embedded in collagen gels. J Cell Physiol 179(2):142-148

25. Kellgren JH, Lawrence JS (1957) Radiological assessment of osteo-arthrosis Ann Rheum Dis 16(4):494-502

26. Kikuchi T, Yamada H, Fujikawa K (2001) Effects of high molecular weight hyaluronan on the distribution and movement of proteoglycan around chondrocytes cultured in alginate beads. Osteoarthr Cartil 9(4):351-356.

27. Kim SH, Djaja YP, Park YB, Park JG, Ko YB, Ha CW (2019) Intra-articular injection of culture-expanded Mesenchymal stem cells without adjuvant surgery in knee osteoarthritis: a systematic review and meta-analysis. Am J Sports Med. 48(11):2839-2849

28. Kim SH, Ha CW, Park YB, Nam E, Lee JE, Lee HJ (2019) Intra-articular injection of mesenchymal stem cells for clinical outcomes and cartilage repair in osteoarthritis of the knee: a meta-analysis of randomized controlled trials. Arch Orthop Trauma Surg 139(7):971-980

29. Kim WB, Alhusayen RO (2015) Skin necrosis from intra-articular hyaluronic acid injection. J Cutan Med Surg 19(2):182-184

30. Kim YS, Choi YJ, Suh DS, Heo DB, Kim Yl, Ryu JS, Koh YG (2015) Mesenchymal stem cell implantation in osteoarthritic knees: is fibrin glue effective as a scaffold? Am J Sports Med 43(1):176-185

31. Kocher MS, Steadman JR, Briggs KK, Sterett WI, Hawkins RJ (2004) Reliability, validity, and responsiveness of the Lysholm knee scale for various chondral disorders of the knee. J Bone Joint Surg Am 86(6):1139-1145

32. Kong L, Zheng LZ, Qin L, Ho KKW (2017) Role of mesenchymal stem cells in osteoarthritis treatment. J Orthop Translat 9:89-103

33. Lamo-Espinosa JM, Mora G, Blanco JF, Granero-Moltó F, Núñez-Córdoba JM López-Elío S, Andreu E, Sánchez-Guijo F, Aquerreta JD, Bondía JM, ValentíAzcárate A, Del Consuelo Del Cañizo M, Villarón EM, Valentí-Nin JR, Prósper F (2018) Intra-articular injection of two different doses of autologous bone marrow mesenchymal stem cells versus hyaluronic acid in the treatment of knee osteoarthritis: long-term follow up of a multicenter randomized controlled clinical trial (phase I/II). J Transl Med 16(1):213

34. Marchal JA, Picon M, Peran M, Bueno C, Jimenez-Navarro M, Carrillo E, Boulaiz H, Rodriguez N, Alvarez P, Menendez P, de Teresa E, Aranega A (2012) Purification and long-term expansion of multipotent endothelial-like cells with potential cardiovascular regeneration. Stem Cells Dev 21(4):562-574

35. Matas J, Orrego M, Amenabar D, Infante C, Tapia-Limonchi R, Cadiz MI, Alcayaga-Miranda F, Gonzalez PL, Muse E, Khoury M, Figueroa FE, Espinoza F (2019) Umbilical cord-derived Mesenchymal stromal cells (MSCs) for knee osteoarthritis: repeated MSC dosing is superior to a single MSC dose and to hyaluronic acid in a controlled randomized phase I/II trial. Stem Cells Trans| Med 8(3):215-224

36. Mazzuca SA, Brandt KD, Dieppe PA, Doherty M, Katz BP, Lane KA (2001) Effect of alignment of the medial tibial plateau and $\mathrm{x}$-ray beam on apparent progression of osteoarthritis in the standing anteroposterior knee radiograph. Arthritis Rheum 44(8):1786-1794

37. Mobasheri A, Batt M (2016) An update on the pathophysiology of osteoarthritis. Ann Phys Rehabil Med 59(5-6):333-339

38. Moreland LW (2003) Intra-articular hyaluronan (hyaluronic acid) and hylans for the treatment of osteoarthritis: mechanisms of action. Arthritis Res Ther 5(2):54-67

39. Morris EA, Wilcon S, Treadwell BV (1992) Inhibition of interleukin 1-mediated proteoglycan degradation in bovine articular cartilage explants by addition of sodium hyaluronate. Am J Vet Res 53(11):1977-1982

40. Nicholls MA, Fierlinger A, Niazi F, Bhandari M (2017) The disease-modifying effects of Hyaluronan in the osteoarthritic disease state. Clin Med Insights Arthritis Musculoskelet Disord 10:1179544117723611

41. Ong KL, Anderson AF, Niazi F, Fierlinger AL, Kurtz SM, Altman RD (2016) Hyaluronic acid injections in Medicare knee osteoarthritis patients are associated with longer time to knee Arthroplasty. J Arthroplast 31(8):1667-1673

42. Orozco L, Munar A, Soler R, Alberca M, Soler F, Huguet M, Sentis J, Sanchez A, Garcia-Sancho J (2013) Treatment of knee osteoarthritis with autologous mesenchymal stem cells: a pilot study. Transplantation 95(12):1535-1541

43. Pers YM, Ruiz M, Noel D, Jorgensen C (2015) Mesenchymal stem cells for the management of inflammation in osteoarthritis: state of the art and perspectives. Osteoarthr Cartil 23(11):2027-2035

44. Pozo MA, Balazs EA, Belmonte C (1997) Reduction of sensory responses to passive movements of inflamed knee joints by hylan, a hyaluronan derivative. Exp Brain Res 116(1):3-9

45. Prockop DJ (1997) Marrow stromal cells as stem cells for nonhematopoietic tissues. Science 276(5309):71-74

46. Roos EM, Roos HP, Lohmander LS, Ekdahl C, Beynnon BD (1998) Knee injury and osteoarthritis outcome score (KOOS)--development of a selfadministered outcome measure. J Orthop Sports Phys Ther 28(2):88-96

47. Rutjes AW, Juni P, da Costa BR, Trelle S, Nuesch E, Reichenbach S (2012) Viscosupplementation for osteoarthritis of the knee: a systematic review and meta-analysis. Ann Intern Med 157(3):180-191

48. Shimizu M, Higuchi H, Takagishi K, Shinozaki T, Kobayashi T (2010) Clinical and biochemical characteristics after intra-articular injection for the treatment of osteoarthritis of the knee: prospective randomized study of sodium hyaluronate and corticosteroid. J Orthop Sci 15(1):51-56

49. Smith MB, Whiteside MG, Campbell DG (1971) The occurrence of heterozygous beta-thalassaemia as screened by quantitative haemoglobin electrophoresis in pregnancy. Med J Aust 1(24):1273-1274 
50. Stove J, Gerlach C, Huch K, Gunther KP, Puhl W, Scharf HP (2002) Effects of hyaluronan on proteoglycan content of osteoarthritic chondrocytes in vitro. J Orthop Res 20(3):551-555

51. Suppan VKL, Tew MM, Wong BC, Chan HK, Chew YW, Tan CS, Nanta Kumar VK, Shafie AA, Sadashiva Rao A (2020) One-year follow-up of efficacy and cost of repeated doses versus single larger dose of intra-articular hyaluronic acid for knee osteoarthritis. J Orthop Surg (Hong Kong) 28(1): 2309499019895029

52. Telikicherla M, Kamath SU (2016) Accuracy of needle placement into the intra-articular space of the knee in osteoarthritis patients for Viscosupplementation. J Clin Diagn Res 10(2):RC15-RC17

53. Tobetto K, Yasui T, Ando T, Hayaishi M, Motohashi N, Shinogi M, Mori I (1992) Inhibitory effects of hyaluronan on [14C] arachidonic acid release from labeled human synovial fibroblasts. Jpn J Pharmacol 60(2):79-84

54. Tung JT, Venta PJ, Caron JP (2002) Inducible nitric oxide expression in equine articular chondrocytes: effects of antiinflammatory compounds. Osteoarthr Cartil 10(1):5-12

55. Turajane T, Chaveewanakorn U, Fongsarun W, Aojanepong J, Papadopoulos KI (2017) Avoidance of Total knee Arthroplasty in early osteoarthritis of the knee with intra-articular implantation of autologous activated peripheral blood stem cells versus hyaluronic acid: a randomized controlled trial with differential effects of growth factor addition. Stem Cells Int 2017:8925132

56. Vezina Audette R, Lavoie-Lamoureux A, Lavoie JP, Laverty S (2013) Inflammatory stimuli differentially modulate the transcription of paracrine signaling molecules of equine bone marrow multipotent mesenchymal stromal cells. Osteoarthr Cartil 21(8):1116-1124

57. Vincent P, Lucas de Couville T, Thomas T (2020) Intra-articular hyaluronic acid for knee osteoarthritis: a Postmarket, open-label, long-term historical control study with analysis detailed per Krellgren-Lawrence radiologic osteoarthritis scale grade. Curr Ther Res Clin Exp 92:100575

58. Vizoso FJ, Eiro N, Cid S, Schneider J, Perez-Fernandez R (2017) Mesenchymal stem cell Secretome: toward cell-free therapeutic strategies in regenerative medicine. Int J Mol Sci 18(9):1852

59. Wang AT, Feng Y, Jia HH, Zhao M, Yu H (2019) Application of mesenchymal stem cell therapy for the treatment of osteoarthritis of the knee: a concise review. World J Stem Cells 11(4):222-235

60. Wu L, Prins HJ, Helder MN, van Blitterswijk CA, Karperien M (2012) Trophic effects of mesenchymal stem cells in chondrocyte co-cultures are independent of culture conditions and cell sources. Tissue Eng Part A 18(15-16):1542-1551

61. Xia P, Wang X, Lin Q, Li X (2015) Efficacy of mesenchymal stem cells injection for the management of knee osteoarthritis: a systematic review and meta-analysis. Int Orthop 39(12):2363-2372

62. Yokota N, Hattori M, Ohtsuru T, Otsuji M, Lyman S, Shimomura K, Nakamura N (2019) Comparative clinical outcomes after intra-articular injection with adipose-derived cultured stem cells or noncultured stromal vascular fraction for the treatment of knee osteoarthritis. Am J Sports Med 47(11):2577-2583

63. Zavan B, Ferroni L, Giorgi C, Calo G, Brun P, Cortivo R, Abatangelo G, Pinton $P$ (2013) Hyaluronic acid induces activation of the kappa-opioid receptor. PLoS One 8(1):e55510

64. Zhang X, Wu S, Naccarato T, Prakash-Damani M, Chou Y, Chu CQ, Zhu Y (2017) Regeneration of hyaline-like cartilage in situ with SOX9 stimulation of bone marrow-derived mesenchymal stem cells. PLoS One 12(6):e0180138

65. Zuk PA, Zhu M, Mizuno H, Huang J, Futrell JW, Katz AJ, Benhaim P, Lorenz HP, Hedrick MH (2001) Multilineage cells from human adipose tissue: implications for cell-based therapies. Tissue Eng 7(2):211-228

\section{Publisher's Note}

Springer Nature remains neutral with regard to jurisdictional claims in published maps and institutional affiliations.

\section{Submit your manuscript to a SpringerOpen ${ }^{\circ}$ journal and benefit from:}

- Convenient online submission

- Rigorous peer review

- Open access: articles freely available online

- High visibility within the field

- Retaining the copyright to your article

Submit your next manuscript at $\boldsymbol{\nabla}$ springeropen.com 